\title{
Clinical features and surgical management of intracranial meningiomas in the elderly
}

\author{
JUNKOH YAMAMOTO, MAYU TAKAHASHI, MASARU IDEI, YOSHITERU NAKANO, YOSHITERU SOEJIMA, \\ DAISUKE AKIBA, TAKEHIRO KITAGAWA, KUNIHIRO UETA, RYO MIYAOKA and SHIGERU NISHIZAWA
}

\author{
Department of Neurosurgery, University of Occupational and Environmental Health, Kitakyushu 807-8555, Japan
}

Received May 29, 2015; Accepted October 11, 2016

DOI: 10.3892/ol.2017.6174

\begin{abstract}
Meningioma accounts for $\sim 25 \%$ of all primary intracranial neoplasms and the incidence increases with age. Prvios population-based studies demonstrated that the annual incidence of intracranial meningiomas was 1.2-3.1/100,000 population. In particular, the incidence of this disease among the elderly is high. Recently, increased life expectancy and greater use of diagnostic radiological imaging led to an increased incidence in the diagnosis of intracranial meningiomas, both symptomatic and asymptomatic, in the elderly. Thus, neurosurgeons may be increasingly confronted with the management of intracranial meningiomas in the elderly. In practice, it is often difficult for physicians to determine whether traditional surgical resection is the optimal management strategy for intracranial meningiomas in the elderly. However, reported clinical studies about the outcome of surgical resection of intracranial meningiomas in the elderly are limited. Increased risk of mortality and morbidity associated with surgical treatment for intracranial meningiomas in the elderly compared with younger patients have been controversial. In the present study, the clinical features of intracranial meningiomas in 70 consecutive intracranial meningioma patients that underwent surgical treatment at the affiliated hospital of University of Occupational and Environmental Health between 2007 and 2013 were assessed. In addition, patient selection and surgical management of intracranial meningioma in elderly patients was discussed. Preoperative factors, including symptoms, tumor location, tumor size, Karnofsky Performance Scale (KPS) score and American Society of Anesthesiology (ASA) score, and postoperative
\end{abstract}

Correspondence to: Dr Junkoh Yamamoto, Department of Neurosurgery, University of Occupational and Environmental Health, 1-1 Iseigaoka, Yahatanishi-ku, Kitakyushu 807-8555, Japan E-mail: yama9218@med.uoeh-u.ac.jp

Abbreviations: ASA, American Society of Anesthesiology; CT, computed tomography; KPS, Karnofsky performance scale; MRI, magnetic resonance imaging; WHO, World Health Organization

Key words: meningioma, geriatric, skull base surgery, craniotomy factors, including pathological diagnosis, tumor proliferation index (Ki-67), resection rate (Simpson grade), length of hospital stay and discharge destination were retrospectively analyzed in patients aged $\geq 75$ years ( $n=16$; elderly group) and $<75$ years ( $n=54$; younger group). Outcomes were assessed 6 months after surgery. Multivariate logistic regression revealed that tumor resection rate (Simpson grade III-V) was an important predictor of surgical complications (odds ratio, 5.662; 95\% confidence interval, 1.323-24.236; $\mathrm{P}=0.0194)$. Perioperative morbidity was not correlated with age ( $>75$ years), tumor location, tumor size, KPS score or ASA score. Thus, the present study indicated that age is not associated with surgical outcome in elderly meningioma patients. Regardless of patient age, the decision to perform surgical resection should be made on an individual basis wherein tumor characteristics and the general health of the patient are considered.

\section{Introduction}

Meningioma accounts for $25 \%$ of all primary intracranial neoplasms, increasing to $40 \%$ if autopsy data are included, indicating that a number of tumors remain clinically silent $(1,2)$. The rising incidence of this tumor with age, in addition to higher life expectancy and more frequent use of diagnostic imaging, has resulted in increased diagnosis of meningioma in the elderly (1). In particular, brain screening with computed tomography (CT) and magnetic resonance imaging (MRI) is easily performed for nonspecific complaints and 'neurological checkups' are performed frequently in Japan (3). Thus, an increasing number of intracranial meningiomas are identified incidentally $(4,5)$. However, few studies have reported the outcome of surgical resection of intracranial meningiomas in the elderly (6-10). Clinically, it is often difficult for physicians to determine whether traditional surgical resection is the optimal management strategy for meningioma in elderly patients: Due to their aging physiology and multiple comorbidities, elderly patients are potentially at risk of unexpected or even life-threatening surgical complications $(7,8)$. Recent studies reported an increased risk of mortality and morbidity in older patients who underwent surgical treatment for intracranial meningioma $(1,11,12)$, whereas other studies have demonstrated similar mortality and morbidity rates in old and young patients $(8,13,14)$. Thus, patient selection and optimal treatment strategies for intracranial meningiomas in the elderly, 
which must consider patient lifestyle, survival benefits vs. side effects and the potential complications of surgery, including neurological deficits, continue to be debated $(6,7,10,15)$. To standardize the surgical indications for intracranial meningiomas in the elderly, a number of studies have proposed grading systems, which include the clinical-radiological grading system (CRGS) (15), the geriatric scoring system (GSS) (16), Karnofsky performance scale (KPS) score, the American Society of Anesthesiology (ASA) score and the Location of Tumor and Peritumoral Edema grading system (SKALE) (17).

In the present study, the clinical features of intracranial meningiomas in elderly patients who underwent surgical treatment at the affiliated hospital of University of Occupational and Environmental Health (Kitakyushu, Japan) were assessed, and patient selection and the surgical management of intracranial meningioma in the elderly was discussed.

\section{Patients and methods}

This study was approved by the institutional review board of the University of Occupational and Environmental Health. Patient informed consent was waived due to the retrospective nature of the study. A total of 70 consecutive patients with intracranial meningioma who underwent craniotomy for resection of meningiomas between April 2007 and December 2013 were included. All patients were newly diagnosed with intracranial meningiomas. Recurrent cases, previous treatment of the brain with radiotherapy, and patients under 18 years of age were excluded from the study. The clinical diagnosis and treatment decision for all patients were based on the results of CT and MRI. Surgery was indicated for symptomatic patients, as well as asymptomatic patients that exhibited evidence of tumor progression on CT and/or MRI. Patients with no history of epileptic seizures, with the exception of those with posterior fossa tumors, were administered prophylactic anticonvulsant therapy (valproate, $600-800 \mathrm{mg} / \mathrm{day}$ or carbamazepine, 100-200 mg/day) for 2-4 weeks, and this treatment was prolonged for those with a history of epileptic seizures for $\geq 2$ years. During the surgical procedures, a neuronavigation system (Kolibri; BrainLAB, Heimstetten, Germany) and an electrophysiological monitoring system (Neuromaster MEE-1216; Nihon Kohden Corporation, Tokyo, Japan) were used for microsurgical tumor resection. All patients received postoperative care in the intensive care unit at the University of Occupational and Environmental Health and rehabilitation therapy commenced on the first postoperative day.

Patient data was obtained by reviewing admission, surgical and anesthesia records, and patients' postoperative status was determined by reviewing outpatient clinical charts. The following patient data were collected: Age at diagnosis, gender, preoperative factors (patient symptoms, tumor location, maximum tumor size, peritumoral brain edema, KPS score and ASA score), and postoperative factors [pathological diagnosis, tumor proliferation index (Ki-67), tumor resection rate (Simpson grade), length of hospital stay and discharge destinations]. Patient outcomes were assessed 6 months after surgery. Patient data was then retrospectively compared between patients aged $\geq 75$ years $(n=16$; elderly group) and those aged $<75$ years ( $n=54$; younger group). Tumor location was divided into four groups: Convexity, falx, parasagittal and skull base. The maximum tumor size was measured on a contrast-enhanced T1-weighted image prior to surgery. Peritumoral brain edema was measured on preoperative T2-weight images, as described previously (17). Briefly, severe brain edema was defined when the ratio of maximum diameter of edema to the maximum diameter of the tumor was $>1$. Moderate brain edema was defined when this ratio was $\leq 1$. Tumor resection rate was determined according to the Simpson grade (18) and tumors were pathologically graded according to the World Health Organization (WHO) classification (19). In addition, proposed grading scoring systems, including CRGS, GSS, and SKALE, were used to calculate scores for each patient using admission data, according to previous studies $(9,15,17)$ and the correlation between these scores and surgical outcomes was assessed.

Statistical analysis. Differences in perioperative characteristics between the elderly and younger groups were compared using an unpaired $t$-test for binomial data and the Fisher exact test and Mann-Whitney $U$ test were used for the comparison of nonparametric data. Multiple logistic regression analysis was performed to determine the association between the various risk factors for perioperative surgical complications [age ( $\geq 75$ years), tumor location (skull base), maximum tumor size ( $\geq 5 \mathrm{~cm}$ diameter), preoperative KPS ( $\geq 70 \%$ ) and tumor resection rate (Simpson grade, $\geq$ III)]. Odds ratios and $95 \%$ confidence intervals were calculated for each risk factor. Similarly, risk factors for surgical complications, including proposed grading scoring systems, were evaluated by multiple logistic analyses. $\mathrm{P}<0.05$ was considered to indicate a statistically significant difference. All statistical analyses were performed using StatView 5.0 statistical software (SAS Institute, Cary, NC, USA).

\section{Results}

Patients. Patient characteristics, preoperative factors and outcomes are shown in Table I. The mean ( \pm standard deviation) ages of the elderly and younger patient groups were 81.1 \pm 5.3 years (range, 75-92 years) and 60.0 \pm 9.6 years (range, 35-73 years), respectively. The elderly patient group consisted of 4 male and 12 female patients, while the younger group consisted of 10 males and 44 females. Elderly patients most frequently presented with dementia as their initial symptom $(31.3 \%)$, however, this was rare in the younger patient group (3.7\%). Younger patients most commonly presented with visual disturbances $(20.4 \%)$ and cranial nerve disturbances (20.4\%). A total of 19 patients (35.2\%) in the younger group were asymptomatic compared with 1 patient $(6.3 \%)$ in the elderly group.

Preoperative KPS scores were significantly lower in the elderly group compared with the younger group $(\mathrm{P}<0.0001)$. Similarly, preoperative ASA scores were also significantly lower in the elderly group than the younger group $(\mathrm{P}=0.0108)$. Regarding tumor location, parasagittal meningiomas were the most common in elderly patients (37.5\%), however, only 1 case of parasagittal meningioma was observed in the younger patient group $(1.9 \%)$. The majority of tumors in younger patients were located at the skull base $(70.3 \%)$. Notably, tumor size in the elderly group was significantly 
Table I. Clinicopathological characteristics, perioperative factors and outcome of 70 intracranial meningioma patients.

\begin{tabular}{|c|c|c|c|}
\hline Parameter & Elderly patient group, n (\%) & Younger patient group, n (\%) & P-value \\
\hline Age, years & & & $<0.0001$ \\
\hline Mean \pm SD & $81.1 \pm 5.3$ & $60.0 \pm 9.6$ & \\
\hline Range & $75-92$ & $35-73$ & \\
\hline Gender & & & 0.7226 \\
\hline Male & $4(25.0)$ & $10(18.5)$ & \\
\hline Female & $12(75.0)$ & $44(81.5)$ & \\
\hline \multicolumn{4}{|l|}{ Symptoms } \\
\hline Visual disturbances & $2(12.5)$ & $11(20.4)$ & \\
\hline Cranial nerves ${ }^{\mathrm{a}}$ & $2(12.5)$ & $11(20.4)$ & \\
\hline Headache & $1(6.3)$ & $3(5.6)$ & \\
\hline Dementia & $5(31.3)$ & $2(3.7)$ & \\
\hline Hemiparesis & $3(18.8)$ & $2(3.7)$ & \\
\hline Ataxia & $2(12.5)$ & $4(7.4)$ & \\
\hline Epilepsy & $0(0.0)$ & $2(3.7)$ & \\
\hline Asymptomatic & $1(6.3)$ & $19(35.2)$ & \\
\hline Preoperative KPS, \% & & & $<0.0001$ \\
\hline$\geq 80$ & $9(56.2)$ & $52(96.2)$ & \\
\hline $60-70$ & $1(6.3)$ & $1(1.9)$ & \\
\hline$<50$ & $6(37.5)$ & $1(1.9)$ & \\
\hline Preoperative ASA score & & & 0.0108 \\
\hline Class 1 & $1(6.3)$ & $15(27.7)$ & \\
\hline Class 2 & $12(75)$ & $38(70.4)$ & \\
\hline Class 3 & $3(18.7)$ & $1(1.9)$ & \\
\hline Class 4-5 & $0(0.0)$ & $0(0.0)$ & \\
\hline Tumor location & & & 0.0316 \\
\hline Convexity & $4(25.0)$ & $9(16.7)$ & \\
\hline Falx & $1(6.3)$ & $6(11.1)$ & \\
\hline Parasagittal & $6(37.5)$ & $1(1.9)$ & \\
\hline Skull base & $5(31.2)$ & $38(70.3)$ & \\
\hline Maximum tumor size, $\mathrm{cm}$ & & & 0.0004 \\
\hline Mean \pm SD & $50.1 \pm 13.3$ & $34.9 \pm 14.7$ & \\
\hline Peritumoral edema ${ }^{\mathrm{b}}$ & & & 0.0001 \\
\hline None & $3(18.8)$ & $37(68.5)$ & \\
\hline Moderate & $8(50.0)$ & $15(27.8)$ & \\
\hline Severe & $5(31.2)$ & $2(3.7)$ & \\
\hline Pathological diagnosis & & & 0.0147 \\
\hline Meningothelial & $6(37.5)$ & $41(75.9)$ & \\
\hline Fibrous & $3(18.8)$ & $0(0.0)$ & \\
\hline Transitional & $0(0.0)$ & $3(5.6)$ & \\
\hline Psammomatous & $5(31.2)$ & $8(14.8)$ & \\
\hline Other ${ }^{\mathrm{b}}$ & $2(12.5)$ & $2(3.7)$ & \\
\hline $\mathrm{Ki}-67, \%$ & & & 0.0336 \\
\hline Mean \pm SD & $2.3 \pm 2.5$ & $1.4 \pm 1.0$ & \\
\hline Simpson's grade & & & 0.0792 \\
\hline I & $6(37.5)$ & $15(27.8)$ & \\
\hline II & $8(50.0)$ & $19(35.1)$ & \\
\hline III & $0(0.0)$ & $4(7.4)$ & \\
\hline IV & $2(12.5)$ & $15(27.8)$ & \\
\hline $\mathrm{V}$ & $0(0.0)$ & 1 (1.9) & \\
\hline
\end{tabular}


Table I. Continued.

\begin{tabular}{|c|c|c|c|}
\hline Parameter & Elderly patient group, n (\%) & Younger patient group, n (\%) & P-value \\
\hline Mean \pm SD & $25.7 \pm 8.7$ & $23.6 \pm 14.1$ & \\
\hline Median & 25 & 17 & \\
\hline Discharge destination & & & 0.0420 \\
\hline Home & $11(68.8)$ & $49(90.7)$ & \\
\hline Rehabilitation Center & $5(31.2)$ & $5(9.3)$ & \\
\hline Postoperative mortalities ${ }^{c}$ & $0(0.0)$ & $0(0.0)$ & \\
\hline Postoperative complications & & & 0.1641 \\
\hline Cranial nerve palsy & $1(6.3)$ & $7(13.0)$ & \\
\hline Hemiparesis & $0(0.0)$ & $3(5.6)$ & \\
\hline Speech disturbance & $0(0.0)$ & $1(1.9)$ & \\
\hline Wound infection & $0(0.0)$ & $2(3.7)$ & \\
\hline None & $15(93.8)$ & $41(75.9)$ & \\
\hline
\end{tabular}

Table II. Multivariate logistic regression analysis of factors associated with surgical complications.

\begin{tabular}{lccc}
\hline Parameter & Surgical complication OR & 95\% CI & P-value \\
\hline Age ( $\geq 75$ years) & 0.265 & $0.017-4.071$ & 0.3406 \\
Tumor location (skull base) & 1.504 & $0.244-9.274$ & 0.6598 \\
Maximum tumor size ( $\geq 5 \mathrm{~cm})$ & 4.507 & $0.793-25.603$ & 0.0893 \\
Preoperative KPS ( $\leq 70 \%)$ & 0.814 & $0.050-13.311$ & 0.8854 \\
Severe peritumoral edema & 0.857 & $0.059-12.474$ & 0.9103 \\
Simpson's grade (III-V) & 5.680 & $1.321-24.420$ & 0.0196 \\
\hline
\end{tabular}

OR, odds ratio; CI, confidence interval; KPS, Karnofsky performance scale.

larger than that in the younger group $(\mathrm{P}=0.008)$. In addition, peritumoral brain edema was significantly more severe in the elderly group when compared with the younger group $(\mathrm{P}=0.0001$; Table I).

Pathology. Pathologically, certain differences were observed between the two groups. Elderly patients frequently presented with meningothelial (37.5\%) and psammomatous $(31.2 \%)$ tumor types with a significantly higher proliferation index (Ki-67) than the younger group $(\mathrm{P}=0.05)$. In addition, 1 meningioma (WHO grade II; atypical type) case $(6.3 \%)$ was observed in the elderly group. By contrast, the majority of meningiomas in the younger group were classified as the meningothelial type (75.9\%). One meningioma case (WHO grade II; clear cell) was observed in the younger patient group, the incidence was low (1.9\%) compared with that in the elderly patient group (Table I). However, no significant difference in the incidence of meningiomas was identified between the groups $(\mathrm{P}=0.4075)$.
Surgery and outcome. Gross total resection (Simpson grade I + II) was performed in 87.5 and $62.9 \%$ of the patients in the elderly and younger groups, respectively. No significant difference in the tumor resection rate was identified between the groups $(\mathrm{P}=0.0792)$. Although no significant difference in the length of hospital stay was identified between the groups, elderly patients were more likely to visit a rehabilitation center/ convalescence hospital following discharge when compared with younger patients $(\mathrm{P}=0.042)$. No postoperative mortality was observed in either group.

Surgical complications in the elderly were limited to one case of facial palsy (7.7\%). A total of 13 patients $(25.6 \%)$ in the younger group exhibited surgical complications, which included the following: Cranial nerve palsy [oculomotor $(n=1)$, trochlear $(n=2)$, abducens $(n=1)$, lower cranial $(n=3)]$, hemiparesis $(n=3)$, speech disturbance $(n=1)$ and wound infection $(n=2)$. One case of facial palsy in the elderly group, 2 cases of cranial palsy and 1 case of hemiparesis in the younger group persisted for $>1$ year after surgery. However, no significant 
Table III. CRGS, GSS and SKALE scores of 70 intracranial meningioma patients according to clinicopathological factors.

A, CRGS score

\begin{tabular}{lccc}
\hline & & CRGS score \\
\cline { 2 - 4 } Clinicopathological factor & $1, \mathrm{n}(\%)$ & $2, \mathrm{n}(\%)$ & $3, \mathrm{n}(\%)$ \\
\hline Size of tumor & $8(11)$ & $24(34)$ & $38(55)$ \\
Location of lesion & $42(60)$ & $6(9)$ & $22(31)$ \\
Presence of edema & $15(21)$ & $48(21)$ & $40(58)$ \\
Neurological condition & $2(3)$ & $46(66)$ & $20(28)$ \\
Concomitant disease & $4(6)$ & $25(36)$ & $38(54)$ \\
KPS score & $7(10)$ & $(28)$ \\
\hline
\end{tabular}

B, GSS score

\begin{tabular}{lccr}
\hline & \multicolumn{2}{c}{ GSS score } \\
\cline { 2 - 4 } Clinicopathological factor & $1, \mathrm{n}(\%)$ & $2, \mathrm{n}(\%)$ & $3, \mathrm{n}(\%)$ \\
\hline Tumor size & $18(26)$ & $25(36)$ & $27(38)$ \\
Neurological deficit & $47(67)$ & $2(3)$ & $21(30)$ \\
KPS score & $7(10)$ & $25(36)$ & $38(54)$ \\
Tumor location & $13(19)$ & $17(24)$ & $40(57)$ \\
Peritumoral edema & $15(21)$ & $15(21)$ & $40(58)$ \\
Diabetes mellitus & $1(1)$ & $4(6)$ & $65(93)$ \\
Hypertension & $0(0)$ & $48(54)$ & $32(46)$ \\
Pulmonary disease & $0(0)$ & $4(6)$ & $66(94)$ \\
\hline
\end{tabular}

C, SKALE score

SKALE score

\begin{tabular}{lccc}
\cline { 2 - 4 } Clinicopathological factor & $0, \mathrm{n}(\%)$ & $2, \mathrm{n}(\%)$ & $4, \mathrm{n}(\%)$ \\
\hline Gender & $14(20)$ & $56(80)$ & - \\
KPS score & $7(10)$ & $12(17)$ & $51(73)$ \\
ASA score & $0(0)$ & $3(4)$ & $67(96)$ \\
Tumor location & $47(67)$ & $23(33)$ & - \\
Presence of edema & $7(10)$ & $23(33)$ & $40(57)$
\end{tabular}

CRGS, clinical-radiological grading system; GSS, geriatric grading system; SKALE, Location of Tumor, and Peritumoral Edema; KPS, Karnofsky performance scale; ASA, American Society of Anesthesiology.

difference in the incidence of surgical complications was identified between the two groups $(\mathrm{P}=0.1641)$. Among the 21 cases of Simpson grade III-IV meningiomas, 19 cases $(90 \%)$ were skull base lesions and 2 cases (10\%) were falx lesions. Among these, surgical complications affected 9 cases, including 6 cases of cranial palsy, 2 cases of hemiparesis and 1 case of speech disturbance. The 6 cases of cranial palsy were associated with manipulation of the cranial nerve, which was encased within or had adhered to tumors during the surgery. One case of hemiparesis and 1 case of speech disturbance were associated with postoperative brain edema in young patients. One case of hemiparesis was associated with postoperative cerebral infarction in the area of the lenticulostriate artery that was encased within the tumor. No cases of symptomatic intracavitary hematoma were observed following surgery.

Among the 2 cases of Simpson grade III-IV meningiomas in the elderly group, 1 patient developed postoperative facial palsy due to nerve manipulation during surgery. Multivariate logistic regression analysis identified a significant association between Simpson grade (III-V) and surgical complications. For patients with meningiomas with a low resection rate (Simpson grade III-V), the risk of experiencing surgical complications was 5.662 times higher than that of patients with a higher resection rate (Simpson grade, I-II) tumors (odds ratio, 5.662, 
Table IV. Multivariate logistic regression analysis of the association between proposed grading system scores and surgical complications.

\begin{tabular}{lccc}
\hline Variables & $\begin{array}{c}\text { Surgical } \\
\text { complications OR }\end{array}$ & \multicolumn{1}{c}{$95 \% \mathrm{CI}$} & P-value \\
\hline Age ( $\geq 75$ years $)$ & 0.107 & $0.007-1.527$ & 0.0992 \\
CRGS score & 1.300 & $0.182-9.258$ & 0.7935 \\
GSS score & 7.875 & $0.503-123.276$ & 0.1414 \\
SKALE score & 2.690 & $0.040-179.157$ & 0.6441 \\
\hline
\end{tabular}

CI, confidence interval; CRGS, clinical-radiological grading system; GSS, geriatric grading system; SKALE, Location of Tumor, and Peritumoral Edema; OR, odds ratio.

95\% confidence interval, 1.323 to $24.236, \mathrm{P}=0.0194$ ) (Table II). Age, tumor location, maximum tumor size and preoperative KPS score were not associated with surgical complications.

CRGS, GSS, SKALE scores. Patient characteristics with corresponding CRGS, GSS and SKALE scores, are shown in Table III. Previous studies demonstrated that a poor outcome following surgical treatment for intracranial meningiomas in the elderly was associated with a score $\leq 9, \leq 15$ and $\leq 7$ for the CRGS, GSS and SKALE, respectively (15-17). The cut-off point for postoperative complications was $\leq 9, \leq 15$, and $\leq 7$ for CRGS, GSS, and SKALE, respectively $(9,15,17)$. Multivariate logistic regression analysis revealed no significant associations between patient age ( $\geq 75$ years), lower CRGS ( $\leq 9)$, GSS $(\leq 15)$, and SKALE scores $(\leq 7)$ and surgical complications ( $\mathrm{P}=0.0992, \mathrm{P}=0.7935, \mathrm{P}=0.1414$ and $\mathrm{P}=0.6441$, respectively) (Table IV). In addition, among elderly patients ( $\geq 75$ years), multivariate logistic regression analysis also revealed no significant difference between lower CRGS, GSS, and SKALE scores and surgical complications $(\mathrm{P}=0.9797, \mathrm{P}>0.9999$ and $\mathrm{P}=0.9883$, respectively).

A case of left frontal convexity meningioma in elderly. An 87-year-old healthy and independent woman consulted her local hospital for an examination of a slight hearing disturbance, and a brain tumor was incidentally detected on a CT scan in November 2009. Therefore, the patient consulted the affiliated hospital of University of Occupational and Environmental Health. Neurological examination revealed no deficit. MRI demonstrated an enhanced extra-axial mass without peritumoral brain edema, corresponding to a parasagittal meningioma (Fig. 1A-C). Conservative treatment was selected due to the asymptomatic nature of the meningioma and the age of the patient. Thus, the patient was subjected to close observation using MRI without prophylactic anticonvulsant therapy. However, the patient discontinued undergoing followup MRI examinations 1 year later. Two years after diagnosis, the patient's condition deteriorated, and she consulted the affiliated hospital of University of Occupational and Environmental Health again in March 2011 with the assistance of her family, using a wheelchair. The patient presented with severe dementia and mild right hemiparesis (KPS, 20\%; ASA score, 3). The patient's CRGS, GSS and SKALE scores were 10, 13 and 8, respectively. MRI revealed tumor progression with peritumoral brain edema (Fig. 1D-F). Subsequently, the patient underwent gross total resection of the tumor (Simpson grade II) without any surgical complications (Fig. 2). The pathological diagnosis was transitional meningioma (WHO grade I) (Ki-67, 2\%). Following surgery, the patient's condition gradually improved and she was able to walk at the time of discharge. Follow-up MRI examination 1 year after surgery revealed no evidence of tumor recurrence (Fig. 2A-C) and a KPS score of $90 \%$.

\section{Discussion}

Based on the results of previous autopsy studies, intracranial meningioma is likely to become an increasingly common disease in the elderly population (20-22). The annual incidence of intracranial meningioma among the elderly is estimated to be 8.4/100,000 persons in Manitoba, Canada (23) compared with 1.2-3.1/100,000 persons per year in the general population of Canada (23), USA (24), United Kingdom (25) and Japan (4), as assessed by epidemiological studies. As a result, neurosurgeons are increasingly confronted with the issue of intracranial meningioma management in the elderly. Surgical treatment of intracranial meningiomas in the elderly may be performed, depending on the patient's age and physiology, with consideration of the technical and ethical issues. However, the risk of surgical treatment must be balanced against the morbidity due to tumor growth that is associated with a conservative treatment.

Previous studies have investigated the natural history of intracranial meningiomas (26-30). The growth rate of intracranial meningiomas has been reported as 2.4-5.3 mm per year (27-29). Other studies have reported that hyperintensity on T2-weighted imaging, a non-skull base location and the absence of calcification on imaging are considered positive indicators of tumor growth in intracranial meningiomas $(30,31)$. In addition, a non-skull base location and the absence of calcification correlate with a high tumor proliferation index (MIB-1) in intracranial meningiomas (32-35). To determine the MIB-1 staining index, the number of cells stained positively with MIB-1 and the total number of cells were counted in several representative fields containing $>1,000$ cells, and the ratio was defined as the MIB-1 staining index. Similarly, in the present study, it was demonstrated that intracranial meningiomas in elderly patients frequently exhibited a non-skull base location, significantly large tumor size and a high proliferation index (Ki-67) when compared with younger meningioma patients. Furthermore, peritumoral edema in intracranial meningiomas was more severe in elderly patients than younger patients. By contrast, previous studies have reported that untreated meningiomas in elderly patients were associated with a significantly lower incidence of tumor growth compared with that in younger patients $(29,31,36)$.

Conservative treatment is usually recommended for asymptomatic meningiomas in the elderly. However, the Brain Tumor Registry of Japan reported that the incidence of surgical WHO grade II and III meningioma cases is 13.3 and $9.4 \%$ in elderly ( $\geq 75$ years old) and younger patients ( $<75$ years old), respectively (37). The present study demonstrated that 

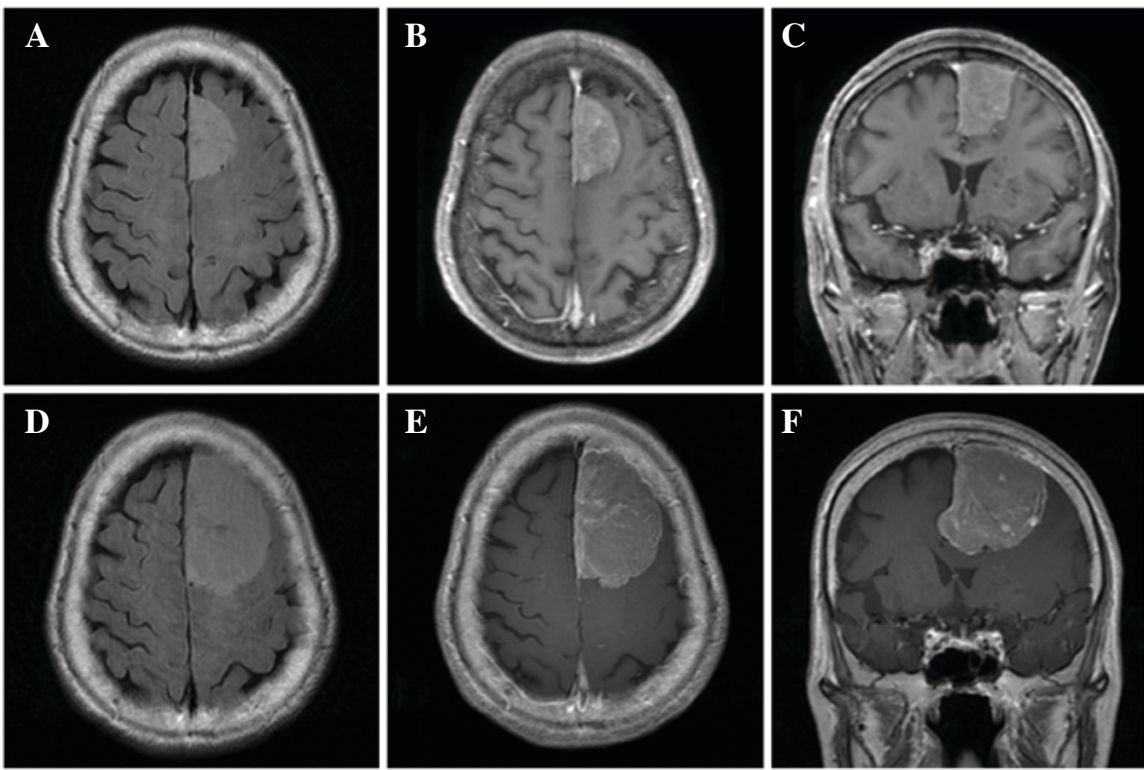

Figure 1. Representative case. (A) Axial fluid-attenuated inversion recovery MRI image and (B) axial and (C) coronal MRI performed at initial examination revealed an enhanced extra-axial mass without peritumoral brain edema. (D) Axial fluid-attenuated inversion recovery MRI image and (E) axial and (F) coronal MRI performed 2 years after diagnosis. MRI revealed evident tumor growth with peritumoral brain edema. MRI, magnetic resonance imaging.
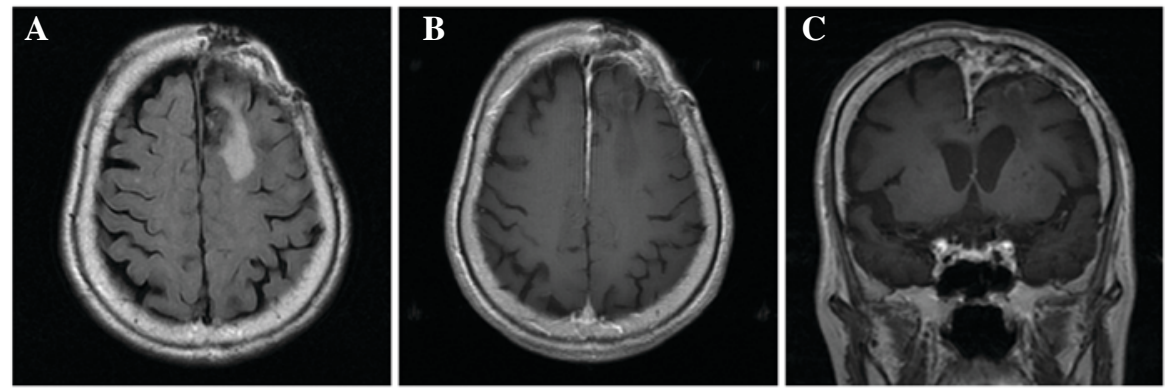

Figure 2. Follow-up magnetic resonance imaging performed 1 year after surgery. (A) Axial view of fluid-attenuated inversion recovery images, (B) axial and (C) coronal views of a contrast-enhanced T1-weighted images. No tumor recurrence was identified.

meningiomas in the elderly exhibit significant histological variations and a higher incidence of WHO grade II (6.5\%) meningiomas when compared with younger meningioma patients. Taken together, these results indicate that surgically treated cases of intracranial meningioma in elderly patients may be biologically different when compared with untreated cases. Therefore, it is suggested that not only symptomatic tumors, but also asymptomatic cases that exhibit tumor growth during follow-up, should be considered for surgical treatment.

Whether there is an increased surgical risk for meningioma in elderly patients remains controversial $(6,7,10,11,17)$. In the present study, the 1-year postoperative mortality rate of meningioma patients was $0 \%$ and the rate of postoperative complications in elderly patients was $7.7 \%$. Multivariate logistic regression analysis indicated that the resection rate (Simpson grade III-V) was an important predictor of postoperative complications. By contrast, surgical complications did not correlate with age ( $>75$ years), tumor location (skull base), tumor size $(>5 \mathrm{~cm})$, preoperative KPS score $(\leq 70 \%)$ or preoperative ASA score (>class 3). Among the Simpson grade III-IV meningioma cases, surgical complications were observed in only 9 cases. One case was observed in the elderly patient group, whereby a facial nerve was encased in perto-clival meningioma. These complications were the result of surgical procedures, including nerve manipulation, postoperative brain edema and postoperative cerebral infarction due to brain retraction and coagulation of perforating arteries. No cases exhibited critical physical complications, such as severe cardiopulmonary dysfunction. Regardless of patient age, surgical difficulties during tumor resection in intracranial meningiomas may lead to postoperative complications and consequently result in a low rate of tumor resection (Simpson grade III-IV).

Previous studies have reported that the 30 day postoperative mortality rate is $0-10.8 \%$ in elderly meningioma patients $(10,15,17,38)$. A recent prospective study of surgical resection of intracranial meningiomas reported that elderly patients ( $>70$ years) exhibited significantly higher 30 -day postoperative mortality rates $(12.0 \%)$ than younger patients (4.6\%) (6). In addition, multiple regression analysis identified age ( $>70$ years), functional health status (including ASA score), preoperative disseminated cancer and tumor location 
(infratentorial) as important predictors of 30-day postoperative mortality (6). In particular, the risk of mortality in elderly patients ( $>70$ years) was 3 times higher than that of younger patients (6). By contrast, a recent systematic meta-analysis indicated that the 1-year mortality rate following meningioma resection in elderly patients was $6-16 \%$ compared with $2-18 \%$ in untreated cohorts $(7,39,40)$. In addition, the survival of elderly patients following meningioma resection was similar to that of the general population $(7,39,40)$.

In a previous study, age was not an independent factor for predicting surgical outcome $(7,10,15)$; in the present study it was demonstrated that age was not associated with increased surgical risk in elderly patients with intracranial meningioma, which was consistent with previous studies $(7,10,15)$. Several studies have assessed predictors for surgical outcome in elderly meningioma patients using CRGS, GSS and SKALE scoring systems that include tumor size, gender, KPS score, ASA score, tumor location, peritumoral edema and concomitant disease $(9,10,16)$. The CRGS, GSS and SKALE scoring systems have been proposed for use in patients aged over 70, 65 and 80 years, respectively. Although these scores may provide useful information for determining the optimal treatment for intracranial meningiomas in the elderly, in actual practice, the difficulties encountered during surgery for intracranial meningiomas (including tumor vascularity, venous drainage, tumor attachment, involvement of cranial nerves and degree of brain stem adhesion or compression) and the surgeon's experience are more likely to affect the surgical outcome (40-42).

A previous study investigated postoperative outcomes in intracranial meningiomas, extending the CRGS and SKALE scoring systems to younger patients ( $\geq 65$ years old) (10). However, they were unable to reproduce the utility of the two proposed grading systems (17). The present study also evaluated surgical complications, using these scoring systems, including GSS. Similarly, no correlation was identified between these scoring systems and surgical complications in all patients, including the elderly. In the present study, only the tumor resection rate was associated with postoperative complications. Thus, the difficulty of meningioma resection may affect the tumor resection rate and consequently lead to postoperative complications. Taken together, for the surgical management of meningioma in the elderly, individual patient health status and characteristics of the tumor should be considered rather than patient age.

An important limitation of this study is that no neuropsychological evaluations were performed. In this study, 5 patients presented with dementia as the initial symptom. The incidence of large tumors, convexity, and falx meningioma was increased in the elderly when compared with younger patients. In elderly intracranial meningioma patients that develop dementia, it is difficult to determine whether the disease is a result of meningiomas or the natural aging process. Thus, prospective studies of surgical management of intracranial meningiomas in the elderly which investigate neuropsychological function, are required.

Neurosurgeons may be increasingly confronted with the issue of intracranial meningioma management in the elderly, which in the majority of cases is treated conservatively. Although the sample number was limited in the present study, it was demonstrated that only tumor resection rate, not patient age, was associated with surgical outcome. If tumors in elderly patients are symptomatic, or asymptomatic with tumor growth during follow-up, specific treatment, including surgical resection, is required. The present study demonstrated that regardless of patient age, the decision to perform surgical resection should be made on an individual basis whereby tumor characteristics and the general health of the patient are considered.

\section{References}

1. Wiemels J, Wrensch M and Claus EB: Epidemiology and etiology of meningioma. J Neurooncol 99: 307-314, 2010.

2. Kurland LT, Schoenberg BS, Annegers JF, Okazaki H and Molgaard CA: The incidence of primary intracranial neoplasms in Rochester, Minnesota, 1935-1977. Ann NY Acad Sci 381: 6-16, 1982

3. Matsutani M: Treatment for asymptomatic meningioma. No To Shinkei 53: 327-330, 2001 (In Japanese).

4. Kuratsu J, Kochi M and Ushio Y: Incidence and clinical features of asymptomatic meningiomas. J Neurosurg 92: 766-770, 2000.

5. Onizuka M, Suyama K, Shibayama A, Hiura T, Horie N and Miyazaki H: Asymptomatic brain tumor detected at brain check-up. Neurol Med Chir (Tokyo) 41: 431-435, 2001.

6. Patil CG, Veeravagu A, Lad SP and Boakye M: Craniotomy for resection of meningioma in the elderly: A multicentre, prospective analysis from the National Surgical Quality Improvement Program. J Neurol Neurosurg Psychiatry 81: 502-505, 2010.

7. Poon MT, Fung LH, Pu JK and Leung GK: Outcome of elderly patients undergoing intracranial meningioma resection - a systematic review and meta-analysis. Br J Neurosurg 28: 303-309, 2014.

8. Bateman BT, Pile-Spellman J, Gutin PH and Berman MF: Meningioma resection in the elderly: Nationwide inpatient sample, 1998-2002. Neurosurgery 57: 866-872, 2005.

9. Cohen-Inbar O, Soustiel JF and Zaaroor M: Meningiomas in the elderly, the surgical benefit and a new scoring system. Acta Neurochir (Wien) 152: 87-97, 2010.

10. Schul DB, Wolf S, Krammer MJ, Landscheidt JF, Tomasino A and Lumenta CB: Meningioma surgery in the elderly: Outcome and validation of 2 proposed grading score systems. Neurosurgery 70: 555-565, 2012.

11. Black P, Kathiresan S and Chung W: Meningioma surgery in the elderly: A case-control study assessing morbidity and mortality. Acta Neurochir (Wien) 140: 1013-1017, 1998.

12. Maurice-Williams RS and Kitchen ND: Intracranial tumours in the elderly: The effect of age on the outcome of first time surgery for meningiomas. Br J Neurosurg 6: 131-137, 1992.

13. Kuratsu J and Ushio Y: Epidemiological study of primary intracranial tumours in elderly people. J Neurol Neurosurg Psychiatry 63: 116-118, 1997.

14. Papo I: Intracranial meningiomas in the elderly in the CT scan era. Acta Neurochir (Wien) 67: 195-204, 1983.

15. Caroli M, Locatelli M, Prada F, Beretta F, Martinelli-Boneschi F, Campanella R and Arienta C: Surgery for intracranial meningiomas in the elderly: A clinical-radiological grading system as a predictor of outcome. J Neurosurg 102: 290-294, 2005.

16. Cohen-Inbar O, Sviri GE, Soustiel JF and Zaaroor M: The Geriatric Scoring System (GSS) in meningioma patients - validation. Acta Neurochir (Wien) 153: 1501-1508, 2011.

17. Sacko O, Sesay M, Roux FE, Riem T, Grenier B, Liguoro D and Loiseau $\mathrm{H}$ : Intracranial meningioma surgery in the ninth decade of life. Neurosurgery 61: 950-955, 2007.

18. Simpson D: The recurrence of intracranial meningiomas after surgical treatment. J Neurol Neurosurg Psychiatry 20: 22-39, 1957.

19. Perry A, Louis DN, Scheithauer BW, Budka $\mathrm{H}$ and von Deimling A: Meningiomas. In: WHO Classification of Tumours of the Central Nervous System. Louis DN, Ohgaki H, Wiestler OD, and Cavenee WK (eds). 4th edition. IARC Press, Lyon, pp164-172, 2007.

20. Helseth A, Mørk SJ, Johansen A and Tretli S: Neoplasms of the central nervous system in Norway. IV. A population-based epidemiological study of meningiomas. APMIS 97: 646-654, 1989.

21. Nakasu S, Hirano A, Shimura T and Llena JF: Incidental meningiomas in autopsy study. Surg Neurol 27: 319-322, 1987. 
22. Sadetzki S, Modan B, Chetrit A and Freedman L: An iatrogenic epidemic of benign meningioma. Am J Epidemiol 151: 266-272, 2000.

23. Rohringer M, Sutherland GR, Louw DF and Sima AA: Incidence and clinicopathological features of meningioma. J Neurosurg 71: 665-672, 1989.

24. Heshmat MY, Kovi J, Simpson C, Kennedy J and Fan KJ: Neoplasms of the central nervous system. Incidence and population selectivity in the Washington DC, metropolitan area. Cancer 38: 2135-2142, 1976.

25. Barker DJ, Weller RO and Garfield JS: Epidemiology of primary tumours of the brain and spinal cord: A regional survey in southern England. J Neurol Neurosurg Psychiatry 39: 290-296, 1976.

26. Claus EB, Bondy ML, Schildkraut JM, Wiemels JL, Wrensch M and Black PM: Epidemiology of intracranial meningioma. Neurosurgery 57: 1088-1095, 2005.

27. Olivero WC, Lister JR and Elwood PW: The natural history and growth rate of asymptomatic meningiomas: A review of 60 patients. J Neurosurg 83: 222-224, 1995.

28. Niiro M, Yatsushiro K, Nakamura K, Kawahara Y and Kuratsu J: Natural history of elderly patients with asymptomatic meningiomas. J Neurol Neurosurg Psychiatry 68 $25-28,2000$

29. Yoneoka Y, Fujii Y and Tanaka R: Growth of incidental meningiomas. Acta Neurochir (Wien) 142: 507-511, 2000.

30. Hashimoto N, Rabo CS, Okita Y, Kinoshita M, Kagawa N Fujimoto Y, Morii E, Kishima H, Maruno M, Kato A and Yoshimine T: Slower growth of skull base meningiomas compared with non-skull base meningiomas based on volumetric and biological studies. J Neurosurg 116: 574-580, 2012.

31. Hashiba T, Hashimoto N, Izumoto S, Suzuki T, Kagawa N, Maruno M, Kato A and Yoshimine T: Serial volumetric assessment of the natural history and growth pattern of incidentally discovered meningiomas. J Neurosurg 110: 675-684, 2009.

32. Torp SH, Lindboe CF, Grønberg BH,Lydersen S and Sundstrøm S: Prognostic significance of Ki-67/MIB-1 proliferation index in meningiomas. Clin Neuropathol 24: 170-174, 2005.
33. Hus CY, Ho DM, Yang CF and Chiang H: Interobserver reproducibility of MIB-1 labeling index in astrocytic tumors using different counting methods. Mod Pathol 16: 951-957, 2003.

34. Kasuya H, Kubo O, Tanaka M, Amano K, Kato K and Hori T: Clinical and radiological features related to the growth potential of meningioma. Neurosurg Rev 29: 293-297, 2006.

35. McGovern SL, Aldape KD, Munsell MF, Mahajan A, DeMonte F and Woo SY: A comparison of World Health Organization tumor grades at recurrence in patients with non-skull base and skull base meningiomas. J Neurosurg 112: 925-933, 2010.

36. Rubin G, Herscovici Z, Laviv Y, Jackson S and Rappaport ZH: Outcome of untreated meningiomas. Isr Med Assoc J 13: 157-160, 2011.

37. Committee of Brain Tumor Registry of Japan: Report of Brain Tumor Registry of Japan (2001-2004), Vol. 13. Neurol Med Chir (Tokyo) 54: 1-102, 2014

38. D'Andrea G, Roperto R, Caroli E, Crispo F and Ferrante L: Thirty-seven cases of intracranial meningiomas in the ninth decade of life: Our experience and review of the literature. Neurosurgery 56: 956-961, 2005.

39. Cahill KS and Claus EB: Treatment and survival of patients with nonmalignant intracranial meningioma: Results from the Surveillance, Epidemiology and End Results Program of the National Cancer Institute. Clinical article. J Neurosurg 115: 259-267, 2011.

40. Sankila R, Kallio M, Jääskeläinen J and Hakulinen T: Long-term survival of 1986 patients with intracranial meningioma diagnosed from 1953 to 1984 in Finland. Comparison of the observed and expected survival rates in a population-based series. Cancer 70: 1568-1576, 1992.

41. Adachi K, Kawase T, Yoshida K, Yazaki T and Onozuka S: ABC Surgical risk scale for skull base meningioma: A new scoring system for predicting the extent of tumor removal and neurological outcome. Clinical article. J Neurosurg 111: 1053-1061, 2009.

42. Yamamoto J, Kakeda S, Takahashi M, Aoyama Y, Soejima Y, Saito T, Akiba D, Korogi Y and Nishizawa S: Dural attachment of intracranial meningiomas: Evaluation with contrast-enhanced three-dimensional fast imaging with steady-state acquisition (FIESTA) at 3 T. Neuroradiology 53: 413-423, 2011. 